\title{
Data-Driven Method for the Measurement of Thickness/Depth Using Pulsed Eddy Current
}

\author{
Lulu Tian, Yuhua Cheng, Chun Yin, \\ Xuegang Huang, ${ }^{1}$ Bo Zhang, and Libing Bai \\ School of Automation Engineering, University of Electronic Science and Technology of China, \\ No. 2006, Xiyuan Ave, West Hi-Tech Zone, Chengdu, Sichuan 611731, P.R. China \\ ${ }^{1}$ Hypervelocity Aerodynamics Institute, China Aerodynamics Research and Development Center, \\ No. 6, South Section, Second Ring Road, Mianyang, Sichuan 621000, P.R. China
}

(Received March 31, 2017; accepted July 4, 2017)

Keywords: pulsed eddy current, thickness measurement, signal processing, nondestructive evaluation, principal component analysis (PCA)

A data-driven method is used to measure the thickness/subsurface-crack-depth of metal specimens. The measuring system is driven by a pulsed eddy current (PEC), and for every detection process a huge data set is obtained because of the high detection speed. To choose the best data to compute the thickness/subsurface-crack-depth, the Jarque-Bera (JB) statistical test is imported into the algorithm. The proposed method is applied to select the best data from the huge detection data set by utilizing the statistical characteristics of the data. Influenced by noise, the detection results may differ when the same thickness/subsurface-crack-depth is measured. To guarantee the accuracy of the measurement, principal component analysis (PCA) is used to combine all detection data. Experimental results show the advantages of the proposed method.

\section{Introduction}

Researchers are now more frequently focusing on non-destructive testing (NDT). ${ }^{(1-3)}$ In particular, the use of a pulsed eddy current (PEC) is developing rapidly. It can be applied in the detection and measurement of surface and subsurface defects. ${ }^{(4-6)}$ Compared with the traditional eddy-current testing (ECT), this method offers considerable advantages. ${ }^{(7,8)}$ In the fields of crack detection, thickness measurement, and crack reconstruction, the PEC method plays an important role $^{(9-11)}$ To maintain large components that are difficult for people to maintain, a periodic monitoring method is needed. ${ }^{(12,13)}$ Malfunction may often be caused by changes in thickness. Many studies have been done on measuring thickness. The power spectrum density (PSD) in the Fourier transform method has been proposed as a way to detect wall-thinning. ${ }^{(14)}$ In many studies, improving detection devices is emphasized. A differential probe has been designed to detect the thickness of a specimen using the peak value and time-to-zero parameters. ${ }^{(15,16)}$ As a material may be complex - for example, iron - the thickness may be difficult to detect. In crack research, iron is sensitive to an induced magnetic field. However, aluminum is not. Consequently, the peak value and time-to-zero parameters are used to detect the thickness of an aluminum plate. ${ }^{(4)}$ Rapid progress is being made toward developing a method for thickness measurement. However,

*Corresponding author: e-mail: yinchun.86416@163.com

http://dx.doi.org/10.18494/SAM.2017.1610 
improvements in the accuracy of measurements at the data level are also required. In the actual detection process, the method takes many data points over a short time at the same thickness. Not all data points can be imported into the processing algorithm because the experimental conditions are different, such as the excitation signal and the experimental electromagnetic environment. Therefore, it is important to select useful data to compute the thickness. In the study, statistical tests and the principal component analysis (PCA) method are used to process the data. The poor detection signals can be recognized as different from good detection signals when a similar thickness is not detected; data from poor detection signals deviates from the correct value. Thus, the distribution of a poor signal is very different from that of a correct signal. As the sampling rate is $2 \mathrm{kHz}$ and 2500 points are obtained by the system for every signal, it is sufficient to compute the distribution of the signal. This characteristic can be used to separate the poor signals from the good signals. The Jarque-Bera (JB) statistical test method was proposed in this study to deal with this problem. The skewness and kurtosis of the signals are used in the method. In the JB test, a value is calculated to evaluate whether the signal is poor or good. Many good signals may be selected from a huge number of detected signals. To make the results more accurate, these selected signals are processed by the PCA method. The fusion of these two data-driven methods can improve the accuracy of thickness measurements to a large extent and the results reflect this.

This paper is organized as follows. In Sect. 2, the basic electromagnetic laws and method are presented. In Sect. 3, the experiment setup is shown, and the verification experiment is discussed in Sect. 4. Finally, conclusions are offered in Sect. 5.

\section{Electromagnetic Analysis and Data Processing}

\subsection{Electromagnetic analysis}

The basic principle of the PEC method used in this study is the skin effect. Induced eddy currents contain information on the thickness of the specimen. The equation is ${ }^{(17)}$

$$
\delta=\frac{1}{\sqrt{\pi f \sigma \mu}}
$$

where $\delta$ is the skin depth, $f$ is the excitation signal frequency, $\sigma$ is the electrical conductivity of the material, and $\mu$ is the magnetic permeability of the material. As the skin depth $\delta$ decreases, the frequency $f$ becomes higher when $\mu$ and $\sigma$ are constant.

Figure 1 presents the relationships in the skin effect. The figure shows that the eddy current density is higher when the current is close to the surface. Figure 2 shows the PEC detection platform. When excitation signal $I_{1}$ changes, it produces the magnetic field $B_{1}$. Eddy current $I_{2}$ is induced in the specimen according to Maxwell's laws. Figure 3 shows the simulation of crack detection by the PEC method using the COMSOL software. It presents that the density of induced eddy current is highest under the excitation coil, and the distribution of the induced eddy current density is different when there is a crack under the coil. The probe is designed and optimized based on this theory and method.

For different excitation frequencies $f$ of $I_{1}$, the induced magnetic field $B_{2}$ is different when the skin depth is larger than the specimen thickness. Using the magnetic field $B_{2}$, the thickness can be obtained. When the frequency is high, the skin depth is small. If the skin depth $\delta$ is smaller than 


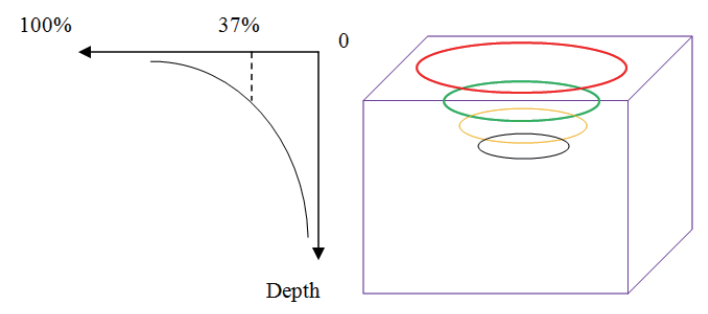

Fig. 1. (Color online) Eddy current skin effect.
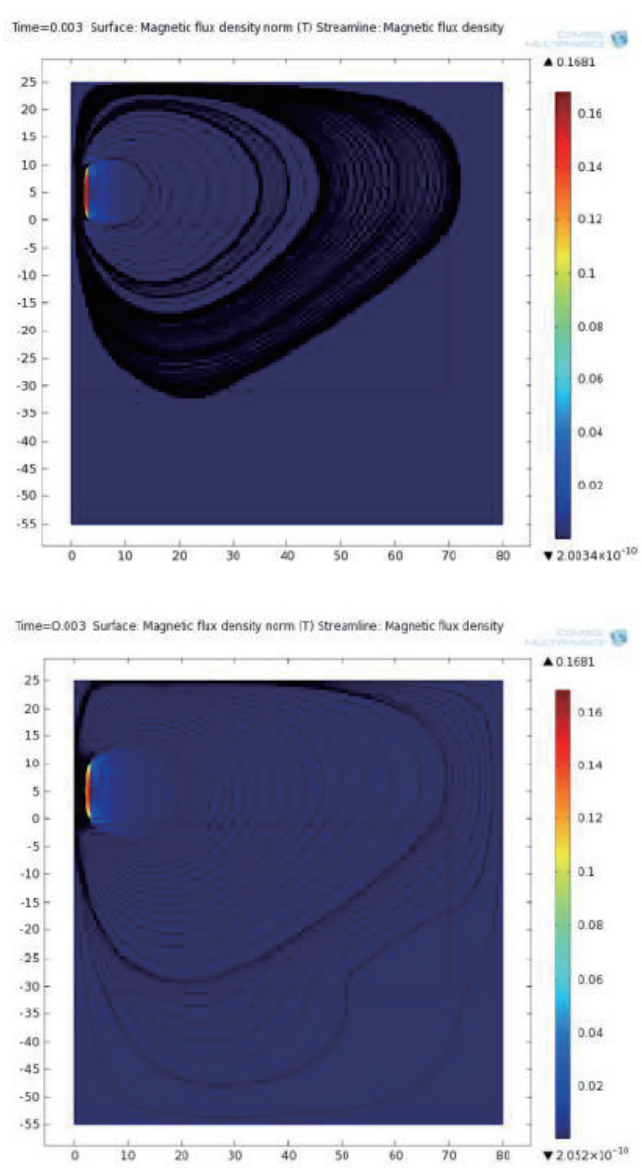

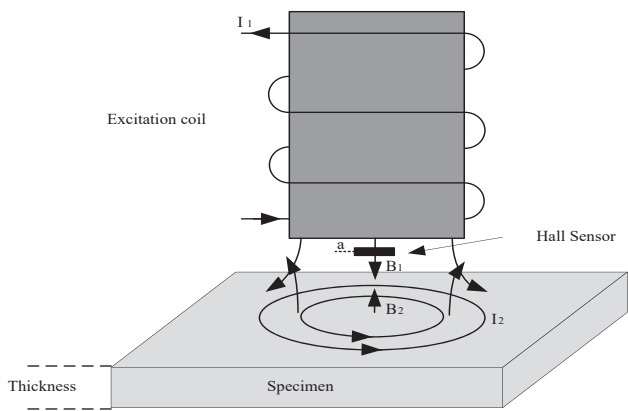

Fig. 2. Eddy current distribution.
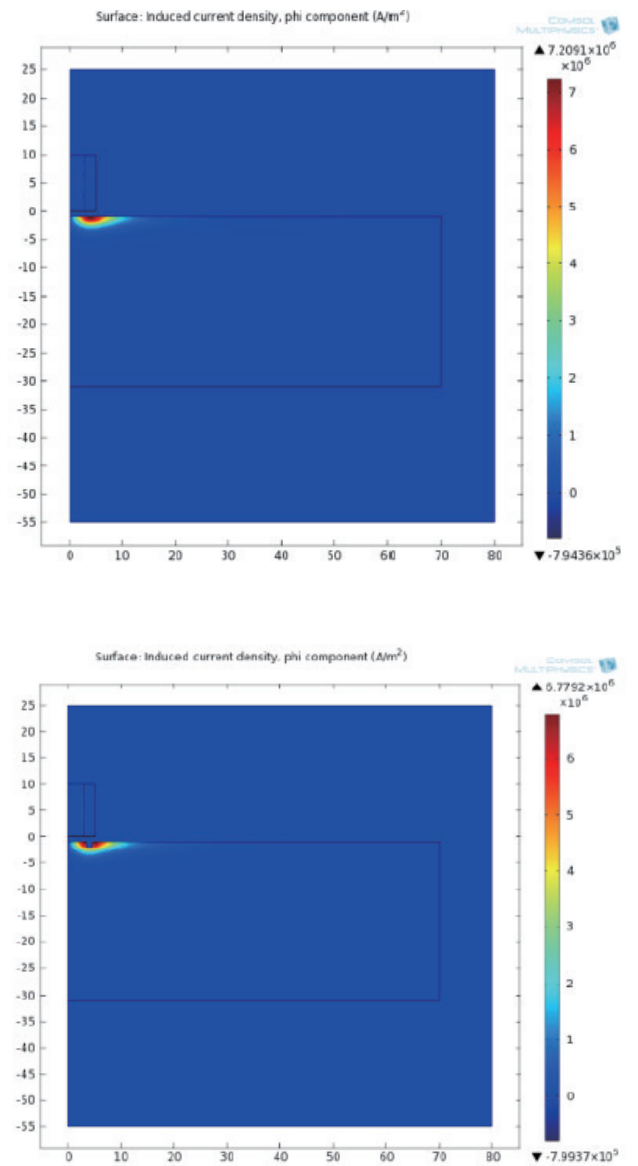

Fig. 3. (Color online) Induced eddy current distribution obtained by simulation.

the specimen thickness, then the magnetic field $B_{2}$ induced by the eddy current will be the same when detecting two different thicknesses, as shown in Fig. 4. However, the skin depth will be different when the frequency is low. This lower frequency is called the mutation frequency, and it is used to compute the thickness. By processing the signal, the frequency can be used to compute the thickness of a metallic material. This method has been evaluated in previous studies. ${ }^{(18)}$ To 


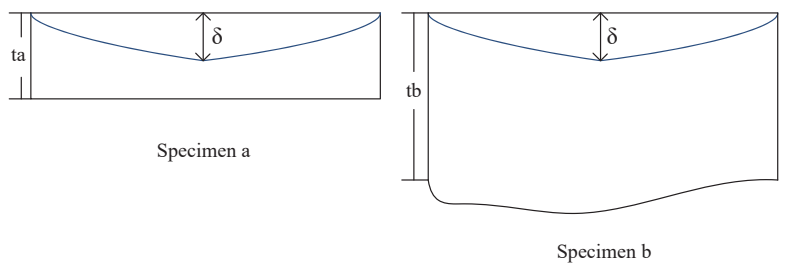

Fig. 4. Skin depth for different thicknesses.

determine the values of the frequency features, a differential parameter $\Delta B(f)$ is defined in Eq. (2):

$$
\Delta B(f)=B_{r e f}(f)-B(f)
$$

where $B_{r e f}$ is the reference signal induced by the eddy current in specimen $b$, and $B$ is the signal induced by specimen $a$.

A Hall sensor is used in this study to detect the signal of the induced magnetic field. Under ideal conditions, the excitation signal is the same during detection. Many features of the response, such as amplitude, time to peak, and time to zero, are used to determine the thickness. ${ }^{(19)}$ Most of these features are easily influenced by noise, such as differences in the excitation signal. In general, one signal is analyzed to calculate the thickness. Consequently, the results often have a large error. To reduce this error, the method should make full use of the detected data from sensors. Furthermore, by applying the features of all the data, one can obtain a more accurate result. For these reasons, we propose a data-driven fusion method in this study. A new data signal can be extracted, and the new data is used in the measurement algorithm.

\subsection{Statistical test method}

The JB test, as a statistical tool, is appropriate to determine the distribution of the data set. It is based on sample kurtosis and the skewness of the data set and assumes a normal distribution. ${ }^{(20,21)}$ For a true normal distribution, the skewness should be near zero and the kurtosis should be three. The JB test involves a chi-square statistical treatment, which determines whether the sample kurtosis and skewness differ markedly from expected values. Based on the sample skewness and kurtosis, a JB test value of the data set can be calculated, and the distribution characteristics can be determined. The distributions of the data set are the same if the JB values are the same. The JB test statistics are presented in this equation:

$$
J B=n\left(\frac{\alpha_{3}^{2}}{6}+\frac{\left(\alpha_{4}-3\right)^{2}}{24}\right)
$$

where $\alpha_{3} \equiv s^{-3} n^{-1} \sum_{i=1}^{n}\left(x_{i}-\bar{x}\right)^{3}, \alpha_{4} \equiv s^{-4} n^{-1} \sum_{i=1}^{n}\left(x_{i}-\bar{x}\right)^{4}, s^{2} \equiv n^{-1} \sum_{i=1}^{n}\left(x_{i}-\bar{x}\right)^{2}, \bar{y}$ is the sample mean, and $s^{2}, \alpha_{3}$, and $\alpha_{4}$ are the second, third, and fourth sample moments, respectively. In the measuring system, there are 2500 sample points for each signal period, which is large enough to determine the statistical distribution. For every different thickness, the system takes multiple measurements and many JB values are calculated using Eq. (3). According to the values, it can be 
determined which data sets should be used in the PCA processing and which sets are incorrect and should be filtered.

\subsection{PCA method}

Figure 5 shows a signal that is processed by the proposed data fusion method. The data fusion method is based on the PCA algorithm. As a statistical tool, PCA is often used to derive the principal components from many data sets of the same kind. The theory of the PCA method is based on the linear combination of the data shown in the following equation:

$$
Y=\omega_{1} X_{1}+\cdots+\omega_{p} X_{p}=\Omega^{\mathrm{T}} X,
$$

where $X$ is the multivariate data, $X_{i}$ is a random variable, and $Y$ is a new random variable. The vector $\Omega$ is selected by the target in Eq. (5),

$$
\max _{\{\Omega:\|\Omega\|=1\}} \operatorname{Var}\left(\Omega^{\mathrm{T}} X\right)=\max _{\{\Omega:\|\Omega\|=1\}} \Omega^{\mathrm{T}} \operatorname{Var}(X) \Omega,
$$

where $\operatorname{Var}(*)$ is the operator of variance. Actually, the vector $\Omega$ is determined by the feature vector $\gamma_{1}$ corresponding to the maximum feature value $\lambda_{1}$ of the variance matrix. For the multivariate matrix data $X, \lambda_{1}$ and $\gamma_{1}$ are the feature value and feature vector if $\lambda_{1}>\lambda_{2}>\ldots>\lambda_{p}$, so $\gamma_{1}^{\mathrm{T}} X$ is the first principal component, then $\gamma_{2}{ }^{\mathrm{T}} X$ is the second principal component, and so on. In the PEC system, every detection signal has the same units and the mean value is zero. This makes the data suitable for PCA.

We assume that $X_{i}$ is the detected data, where $i$ denotes the $i$ th detection. Then the multivariate matrix data can be expressed in Eq. (6):

$$
X=\left[X_{1}, X_{2}, \ldots, X_{p}\right]
$$

Then, using $X$ in the PCA program, a new $p$-dimensional vector set can be obtained, which is arranged according to the contribution value shown in Eq. (7):

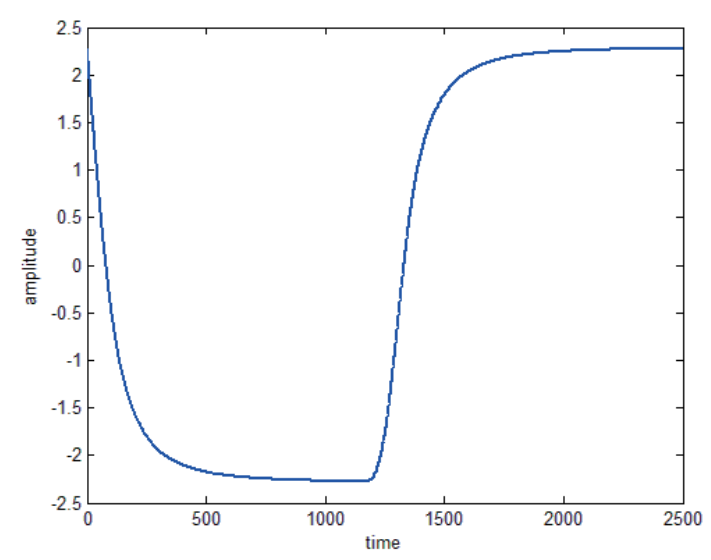

Fig. 5. (Color online) Detection signal. 


$$
\text { Score }=\left[I M F_{1}, I M F_{2}, \ldots, I M F_{p}\right],
$$

where the intrinsic mode function $I M F_{i}$ is the $i$ th principal component data according to PCA. As $X_{i}$ is almost the same as $I M F_{1}$, the contribution to $I M F_{1}$ is above $99 \%$ according to the results. Therefore, $I M F_{1}$ can be used to represent the signals of $X$.

However, $I M F_{1}$ is the data processed by the PCA program, and its value is very different from the source data shown in Fig. 6. This is because the PCA result $I M F_{1}$ is a linear combination of the source data, and this processing changes the amplitude of the result. However, frequency information will not change and this is the reason why the PCA method is suitable for the processing of the PEC signal to obtain information in the frequency domain. Therefore, the main difference is the amplitude, and to make it match the source data, $I M F_{1}$ is transformed into a normalization domain using the mean value of the extreme values of all the source data.

Processed by this method, the new data maintains the main characteristics of the source data and reduce the influences of noise and measuring errors. By fusing with the PCA method, suitable new data may be obtained that can be used in the measuring algorithm.

\section{Experimental Setup}

The experimental platform is shown in Fig. 7(a). A Hall sensor is placed under the excitation core to detect the electromagnetic signal. The detailed structure of the probe is shown in Fig. 7(b).

When detecting thickness, the scanner platform moves on the specimen being probed. The magnetic field detected by the Hall sensor is the signal that is imported into the algorithm.

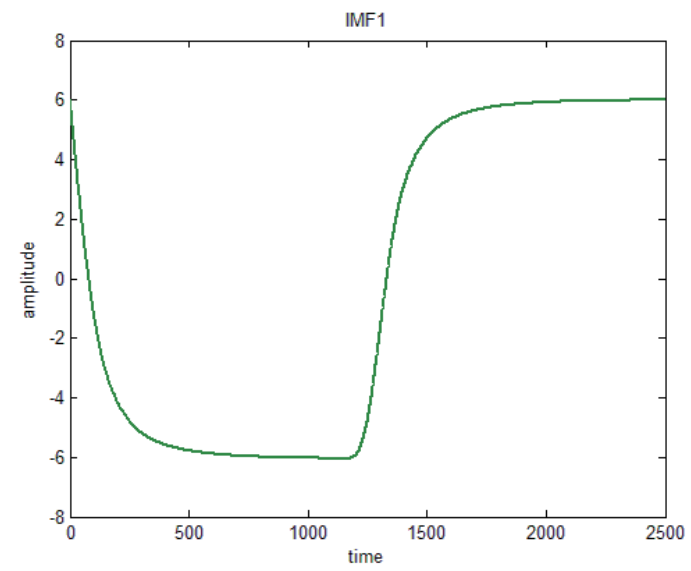

Fig. 6. (Color online) $I M F_{1}$.



(a)

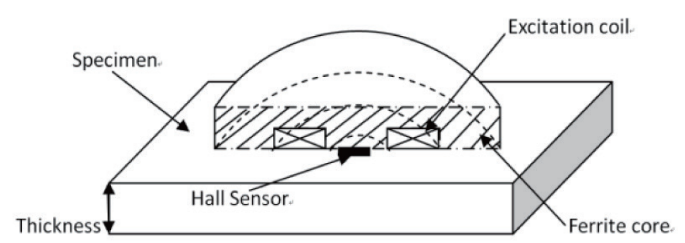

(b)

Fig. 7. (Color online) (a) Experimental device and (b) schematic diagram of the probe. 
The sample shape is shown in Fig. 8. There are ten samples with the thicknesses of 100, 90, 80, $70,60,50,40,30,20$, and $10 \%$ of the original thickness of $10 \mathrm{~mm}$.

\section{Results and Discussion}

In this detection system, the excitation signal is a $200 \mathrm{~Hz}$ square wave and the filter has a cutoff frequency of about $2 \mathrm{kHz}$. The difference value $\Delta B(f)$ is valid when the frequency ranges from $200 \mathrm{~Hz}$ to $2 \mathrm{kHz}$ by FFT. Figure 9 shows the relationship between frequency and $\Delta B$.

Figure 10 shows the relationship between the searching step number and the searching frequency. When the frequency is in the correct range, the searching step number can be used in the algorithm to compute the thickness.

During the experiment, the results when the same thickness is detected more than once are different, as shown in Fig. 11. The figure shows that when detecting a thickness of $2 \mathrm{~mm}$ five times, the mutation frequencies are $1272,1526,1512,1378$, and $1571 \mathrm{~Hz}$, which result in differences in the thickness measurement. Thus, the frequency has to be selected manually for the signal for an accurate result.

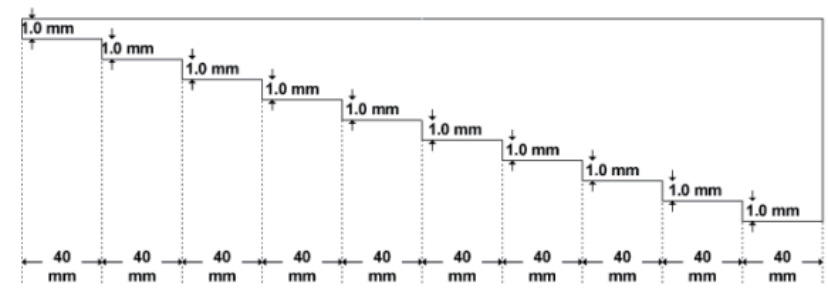

Fig. 8. Samples used in this study.

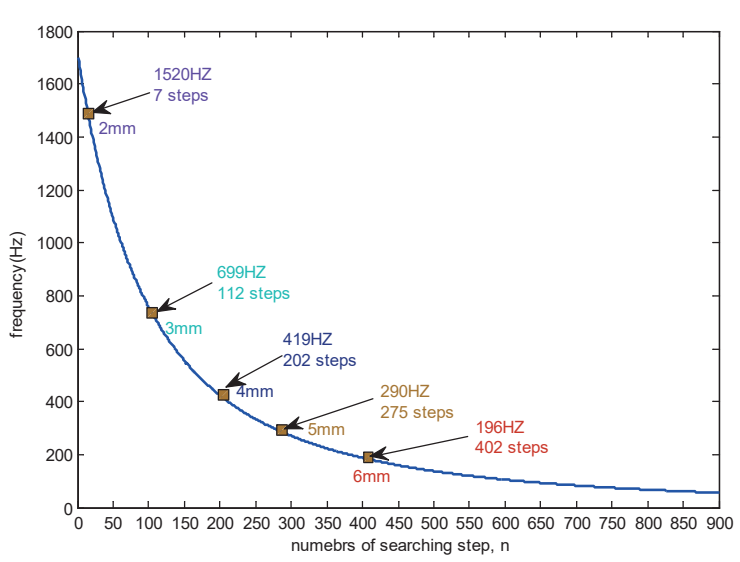

Fig. 10. (Color online) Relationship between searching steps and frequency.

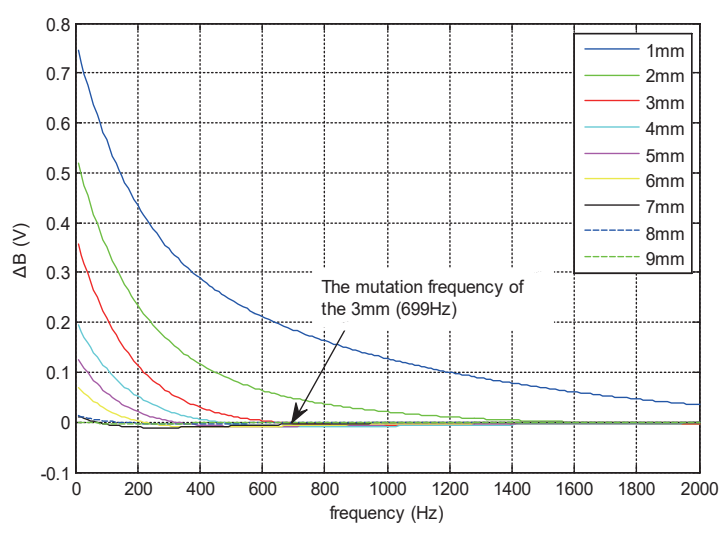

Fig. 9. (Color online) Difference value $\Delta B$ for different frequencies.

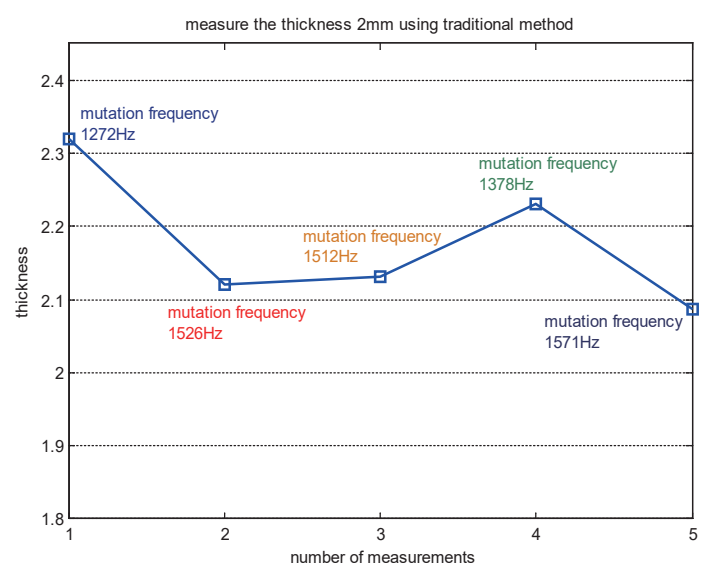

Fig. 11. (Color online) Results for detecting a $2 \mathrm{~mm}$ thickness using a traditional frequency method. 
To solve this problem, the data fusion method is imported into the experiment. First, some good signals should be selected using the following steps:

- Compute the JB test feature values; if the JB values are the same, the signals are correct. Otherwise, the data cannot be used in the algorithm.

- Select five good signals and import them into the PCA algorithm.

- Detect the thickness many times and save the data in a computer.

Figure 12(a) shows the JB test results, which establish that this method could select the best data from the data set. Figure 12(b) shows that the differential value maintains a value of zero when detecting the same thickness. This feature is more stable than the amplitude of JB. In an actual situation, when detecting one thickness, the signals will be identified as effective when the differential value is close to zero. The selected data will be processed by the PCA method. Figure 13 shows a typical result of the data fusion. Figure 13(a) illustrates that the fused data fits the source data very well, and Fig. 13(b) shows that the data is concentrated on the side where more source data is available. The fused data can be used to represent the source data set. For each thickness measurement, the fused data will be collected and imported into the processing program.

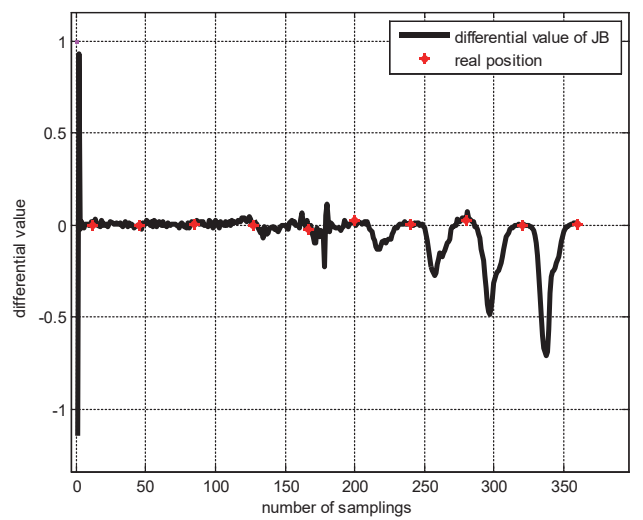

(a)

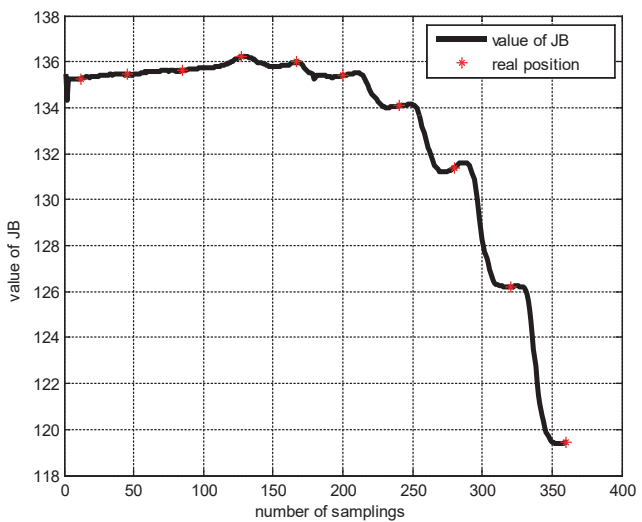

(b)

Fig. 12. (Color online) (a) Results processed by the JB test and (b) differential values of JB.

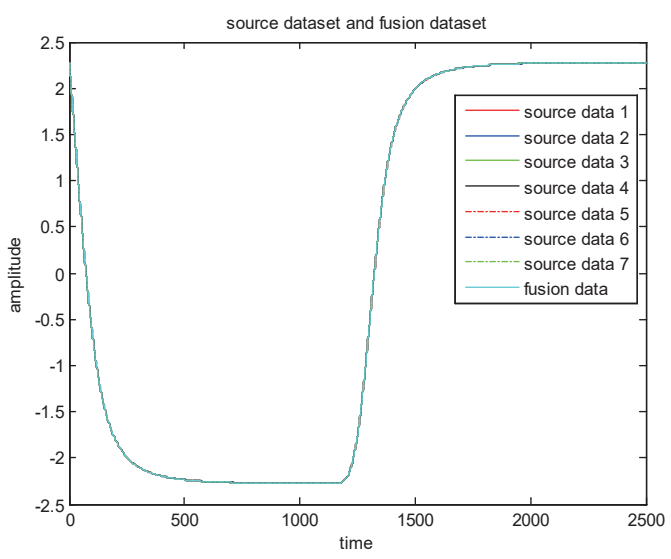

(a)

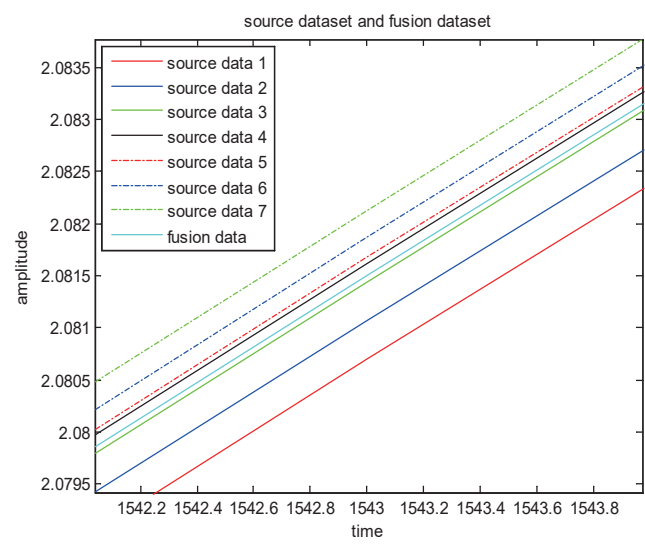

(b)

Fig. 13. (Color online) (a) Full and (b) detailed views of the fused data. 
The method used the five data points selected by the JB test method, and the measurement result is better than any of the five results, as shown in Fig. 14. The error decreases from $0.09 \mathrm{~mm}$, the best of the five results, to $0.08 \mathrm{~mm}$. Furthermore, another experiment was conducted for a 2 $\mathrm{mm}$ thickness, as shown in Fig. 15. The experiment was repeated eight times, and the result was better than that for five measurements; the error was $0.07 \mathrm{~mm}$. The experiments were also carried out for thicknesses of 3, 4, 5, and $6 \mathrm{~mm}$, as shown in Figs. 16-23; the errors were 0.08, 0.06, 0.03, 0.02, $0.05,0.03,0.02$, and $0.03 \mathrm{~mm}$, respectively.

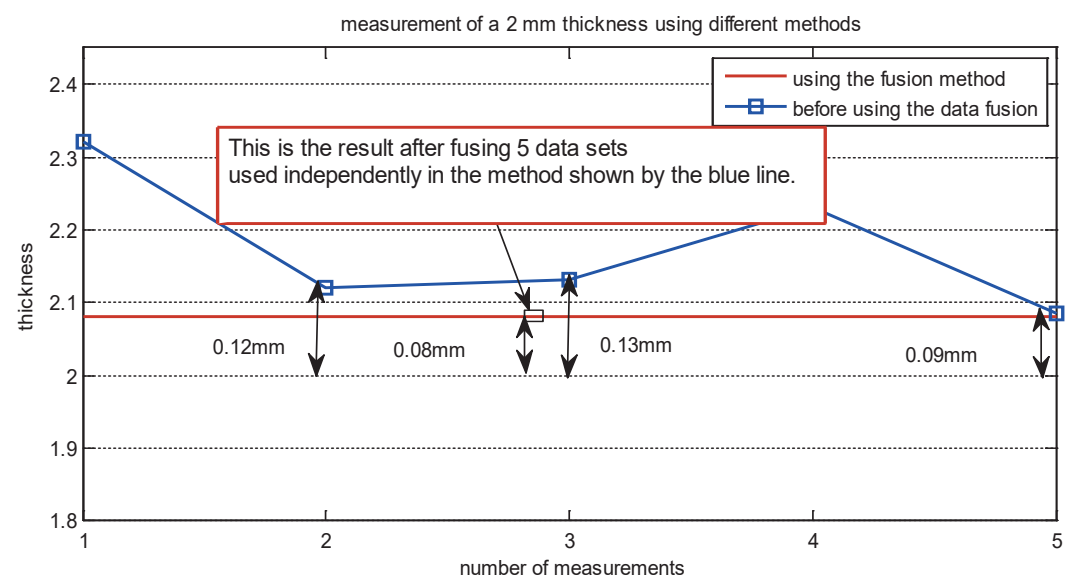

Fig. 14. (Color online) Comparison of the two methods for detecting a $2 \mathrm{~mm}$ thickness five times.

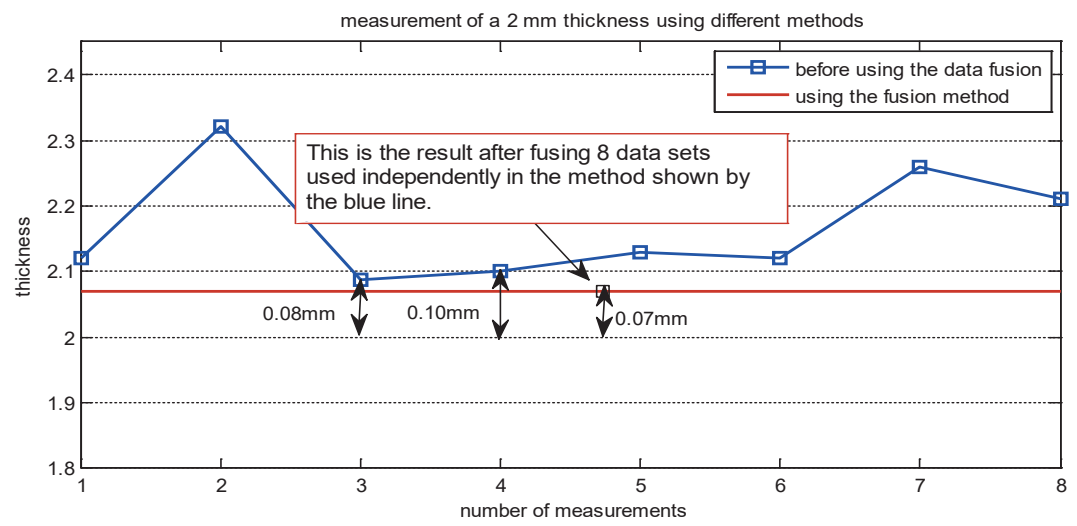

Fig. 15. (Color online) Comparison of the two methods for detecting a $2 \mathrm{~mm}$ thickness eight times.

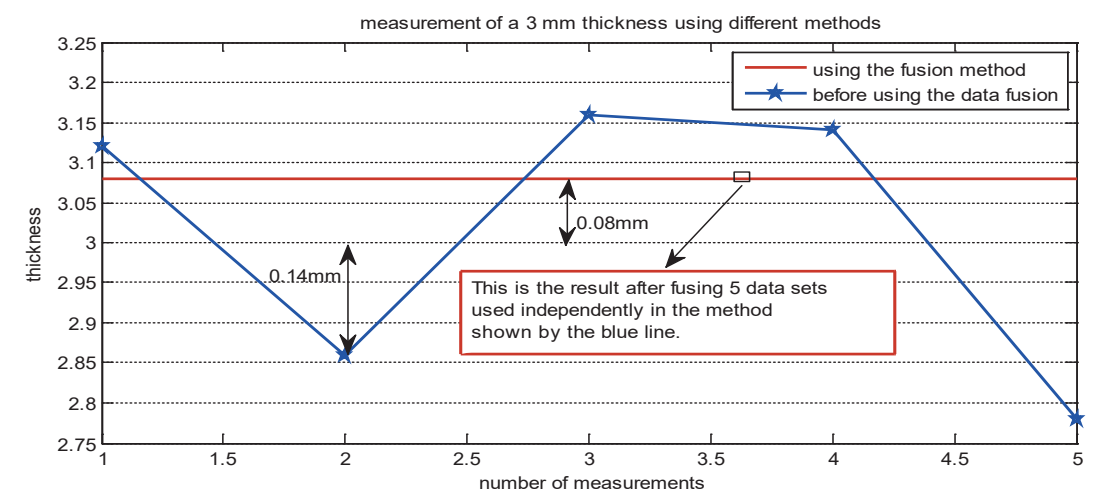

Fig. 16. (Color online) Comparison of the two methods for detecting a $3 \mathrm{~mm}$ thickness five times. 


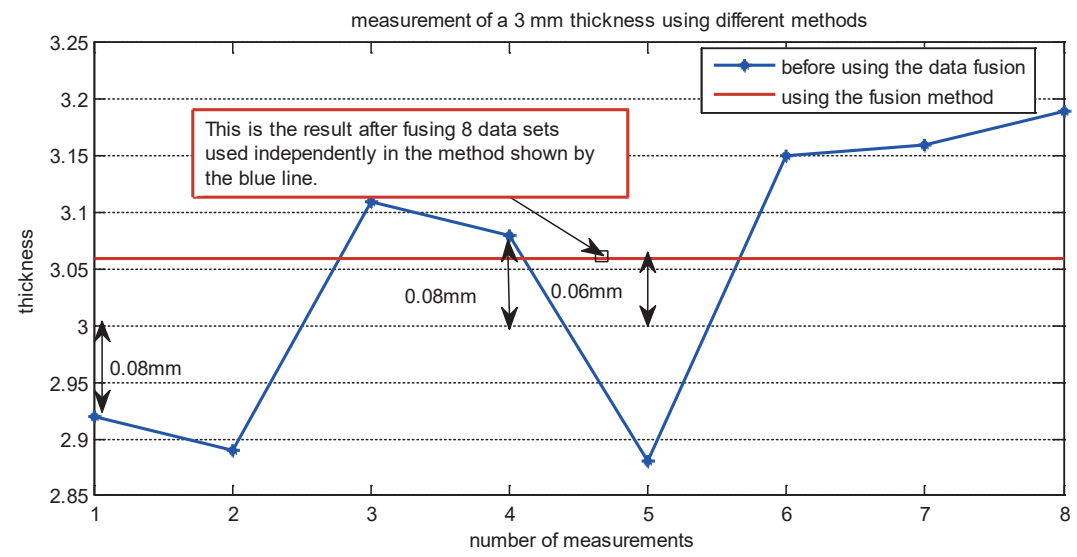

Fig. 17. (Color online) Comparison of the two methods for detecting a $3 \mathrm{~mm}$ thickness eight times.

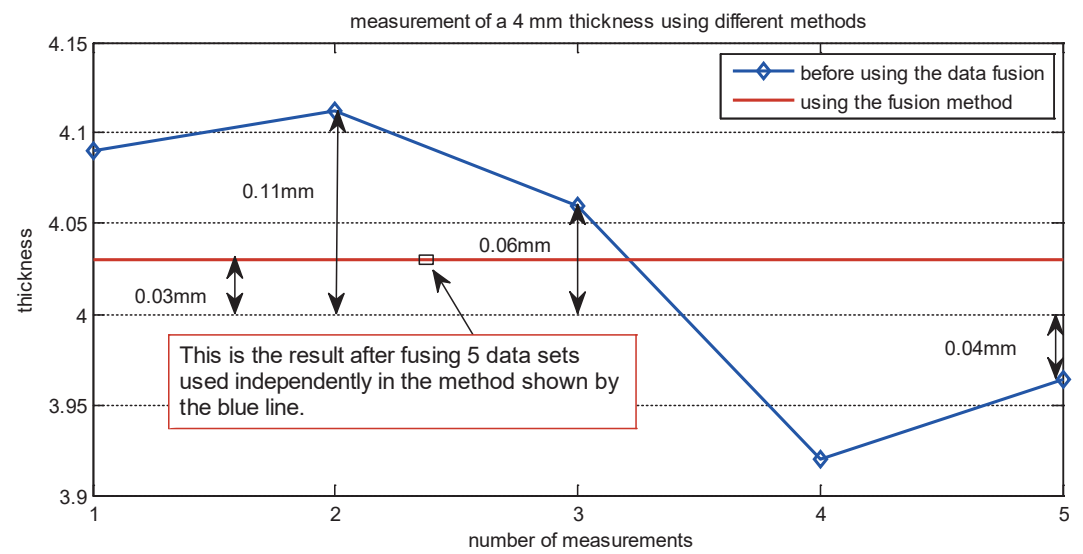

Fig. 18. (Color online) Comparison of the two methods for detecting a $4 \mathrm{~mm}$ thickness five times.

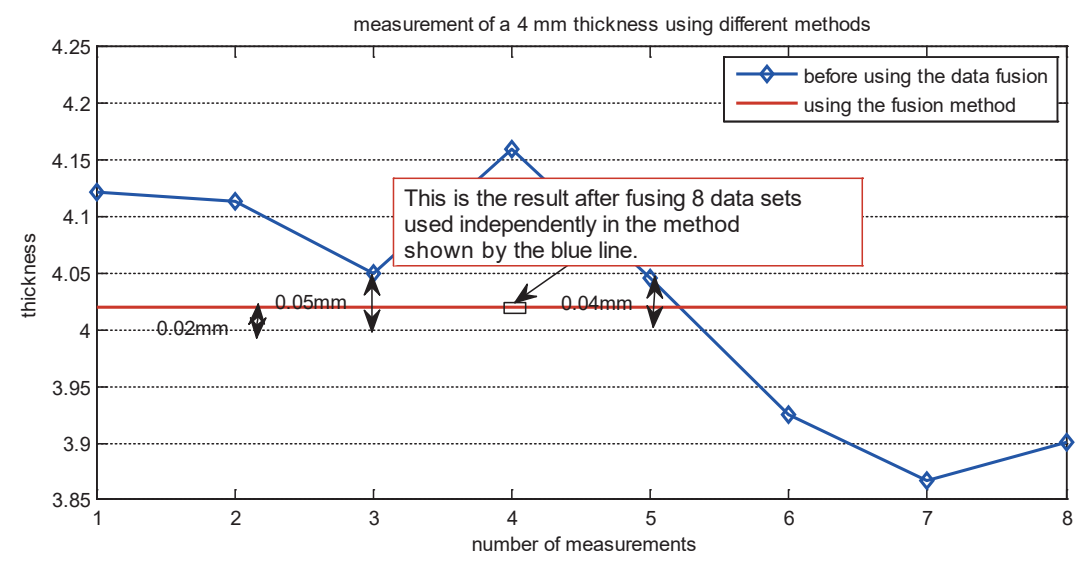

Fig. 19. (Color online) Comparison of the two methods for detecting a $4 \mathrm{~mm}$ thickness eight times.

The results show that the error when using data fusion is slightly smaller than the error when it is not used. From these two results, it can be stated that the results using the fusion method are more accurate on the whole. On the other hand, comparing the two fused results for a five-data-set fusion and an eight-data-set fusion, the result from fusing the eight data sets is better than that for 


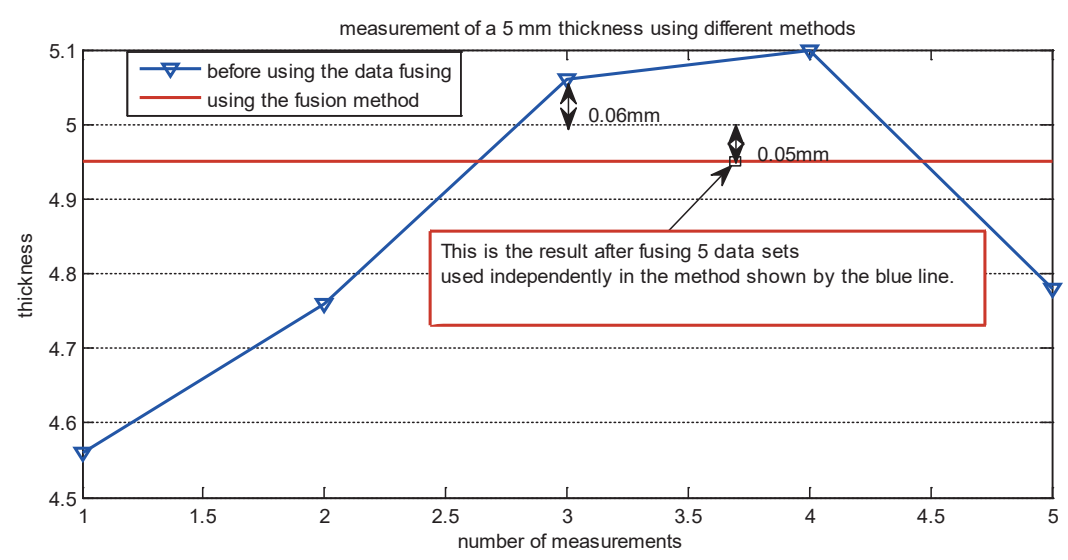

Fig. 20. (Color online) Comparison of the two methods for detecting a $5 \mathrm{~mm}$ thickness five times.

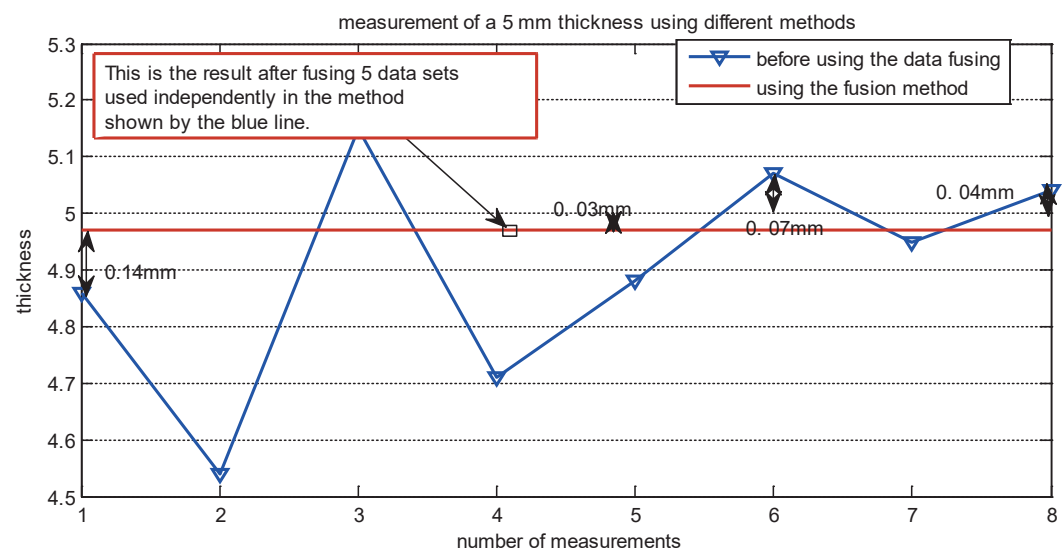

Fig. 21. (Color online) Comparison of the two methods for detecting a $5 \mathrm{~mm}$ thickness eight times.

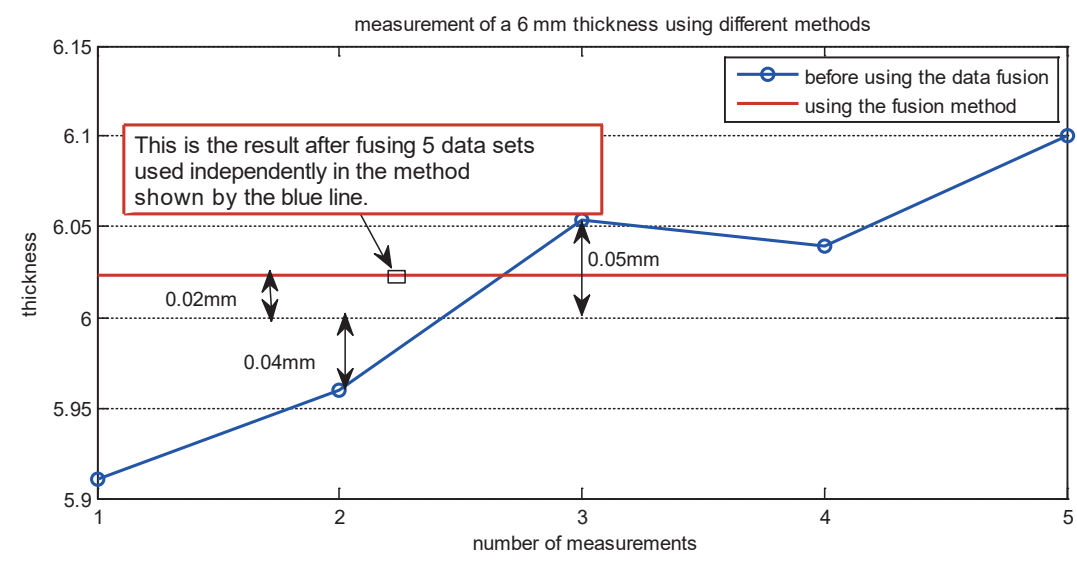

Fig. 22. (Color online) Comparison of the two methods for detecting a $6 \mathrm{~mm}$ thickness five times.

fusing five. The proposed data fusion method can thus improve the measurement accuracy.

For thicknesses of 2, 4, 5, and $6 \mathrm{~mm}$ (as limited by the cutoff frequency; thicknesses over $6 \mathrm{~mm}$ cannot be detected correctly), the mutation frequencies are 1520, 419, 279, and $196 \mathrm{~Hz}$, and the calculated thicknesses are 2.07, 4.02, 4.97, and $6.02 \mathrm{~mm}$, respectively, as shown in Table 1 . 


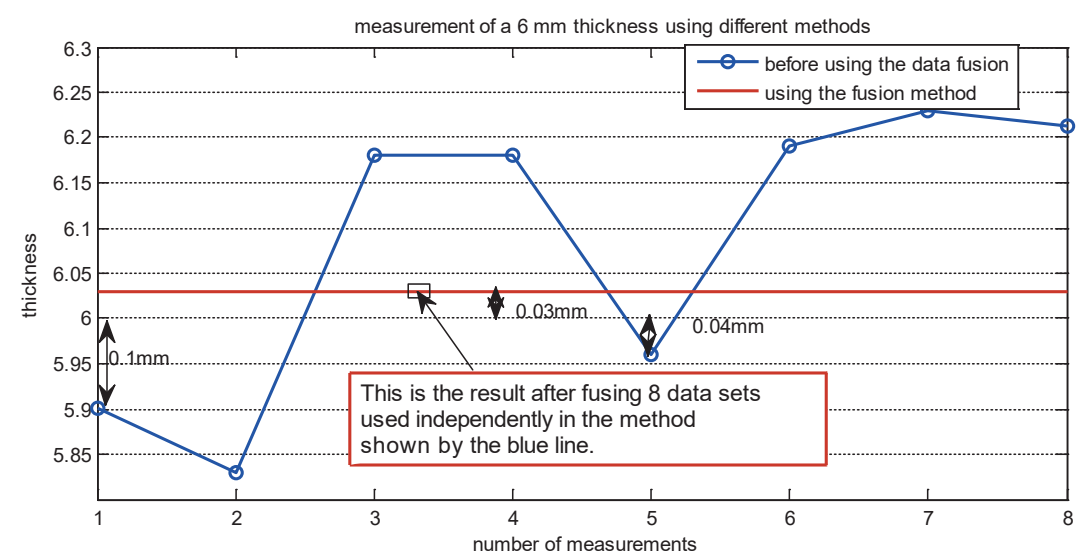

Fig. 23. (Color online) Comparison of the two methods for detecting a $6 \mathrm{~mm}$ thickness eight times.

Table 1

Results of the study.

\begin{tabular}{cccc}
\hline Real thickness $(\mathrm{mm})$ & Mutation frequency $(\mathrm{Hz})$ & Step number & Measured thickness $(\mathrm{mm})$ \\
\hline 2 & 1520 & 7 & 2.07 \\
3 & 699 & 112 & 3.12 \\
4 & 419 & 202 & 4.02 \\
5 & 279 & 275 & 4.97 \\
6 & 196 & 402 & 6.02 \\
\hline
\end{tabular}

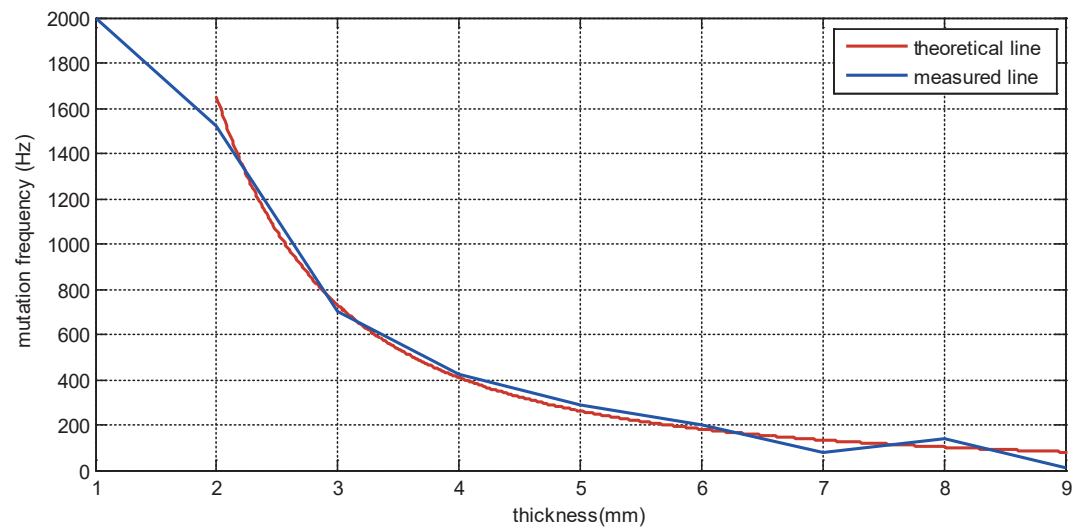

Fig. 24. (Color online) Mutation frequency for different thicknesses.

Figure 24 shows the mutation frequency when the specimen is scanned. It can be seen that the frequency is cut off at $2 \mathrm{kHz}$ when the 1-mm-thick specimen is measured. The frequency should be higher than $2 \mathrm{kHz}$ when the skin depth is less than $1 \mathrm{~mm}$ for this standard detecting material. Additionally, the mutation frequency becomes very unstable when the thickness is more than 6 $\mathrm{mm}$, because the required frequency is lower than $200 \mathrm{~Hz}$. In this system, the mutation frequency can be used to measure thicknesses ranging from 2 to $6 \mathrm{~mm}$, and in this interval, the measured values fit the theoretical values very well. 


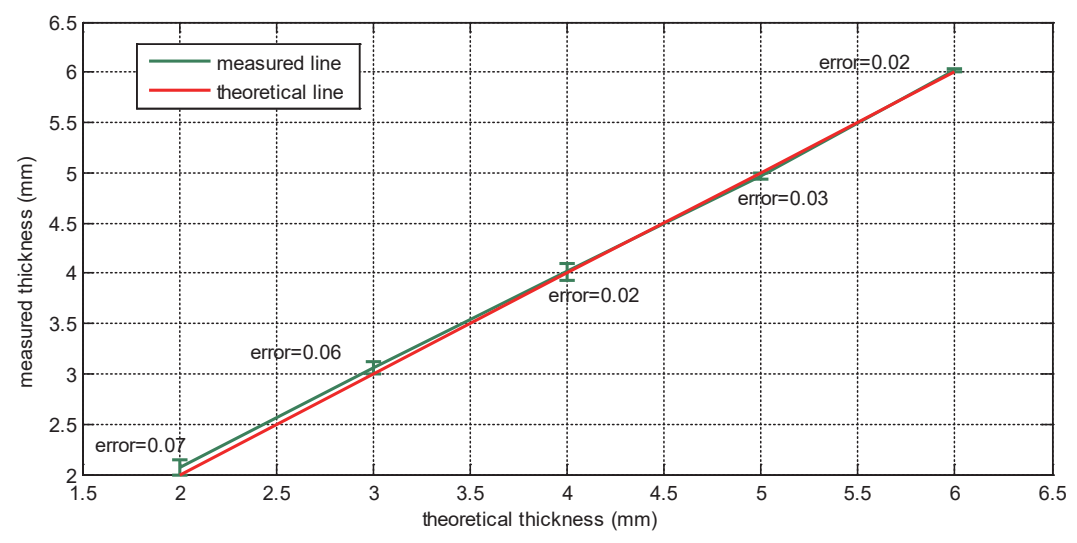

Fig. 25. (Color online) Theoretical thickness vs measured thickness.

Figure 25 shows a comparison between the theoretical thickness and measured thickness values. The largest error is $0.07 \mathrm{~mm}$ and the mean error of the five measured thicknesses is 0.04 $\mathrm{mm}$, which is less than $0.2 \mathrm{~mm}$ in the design shown in previous work. ${ }^{(18)}$

From the results presented here, it can be shown that the measurement results are better than those not processed by the data-driven method. A JB test is used to select the good signals from the huge numbers of detected signals. The entire process is completed by the machine itself. After signal selection, the PCA method is then used. This method reduces the error due to noise while retaining the information in the signal. These two methods in combination make the measurement more accurate.

\section{Conclusions}

In this study, a novel data-driven method for processing a PEC signal was proposed. This method fused the JB test method and the PCA method. As different conditions give rise to the detection, the signals that are induced by the eddy current are very different. To make full use of the signal, the PCA method is used. This method produces a new signal that includes most of the information in the signals. It can reduce the influence of noise and improve the accuracy of the measurement. However, before using the PCA method, good signals must first be obtained. Thus, the JB test method was applied in this study. The distribution regularities of the signals are different between poor signals and good signals, which makes the JB test value different. In general, the good signals predominate. Five signals were selected using this method and were imported into the PCA method. By using these two data-driven methods, the system can select good signals and can reduce the influence of noise. The important point is that the method can improve the accuracy of the thickness measurement to a considerable extent. In future work, this method will be combined with the magneto-optical imaging (MOI) method for subsurface crack detection to visualize subsurface cracks in 3D images.

\section{Acknowledgments}

This work is funded by the National Natural Science Foundation of China (Grant Nos. 61503064, 61671109, and 51407024). 


\section{References}

1 J. Abbaszadeh, H. A. Rahim, R. A. Rahim, and S. Sarafi: Sens. Mater. 25 (2013) 379.

2 Y. He, G. Tian, M. Pan, and D. Chen: Composites Part B 59 (2014) 196.

3 S. Zhang, W. K. Lee, and P. Pong: Sens. Mater. 25 (2013) 423.

4 G. Singh, H. M. Bapat, B. P. Singh, M. Bandyopadhyay, R. K. Puri, and D. N. Badodkar: J. Inst. Eng. (India): Ser. D 94 (2013) 89.

5 C. Yin, S. Dadras, X. Huang, J. Mei, H. Malek, and Y. Cheng: Energy Convers. Manage. 15 (2017) 504.

6 E. Shameli: ASME 2012 Int. Mechanical Engineering Congress and Exposition. (American Society of Mechanical Engineers, November 2012) p. 11.

7 G. Y. Tian and A. Sophian: NDT and E Int. 38 (2005) 77.

8 J. Kim, G. Yang, L. Udpa, and S. Udpa: NDT and E Int. 43 (2010) 141.

9 D. N. Dyck, G. Gilbert, B. Forghani, and J. P. Webb: IEEE Trans. Magn. 40 (2004) 1406.

10 E. Cardelli, A. Faba, and M. Tomassini: IEEE Trans. Magn. 41 (2005) 1616.

11 G. Preda, M. Rebican, and F. I. Hantila: IEEE Trans. Magn. 46 (2010) 3433.

12 J. W. Kim, S. H. Lee, and C. Y. Park: Nucl. Eng. Des. 239 (2009) 2737.

13 K. Takahashi, K. Ando, M. Hisatsune, and K. Hasegawa: Nucl. Eng. Des. 237 (2007) 335.

14 D. G. Park, C. S. Angani, G. D. Kim, C. G. Kim, and Y. M. Cheong: IEEE Trans. Magn. 45 (2009) 3893.

15 C. S. Angani, D. G. Park, C. G. Kim, P. Leela, P. Kollu, and Y. M. Cheong: J. Nondestr. Eval. 29 (2010) 248.

16 C. S. Angani, D. G. Park, G. D. Kim, C. G. Kim, and Y. M. Cheong: J. Appl. Phys. 107 (2010) 09E720.

17 H. J. Krause and M. V. Kreutzbruck: Physica C: Superconductivity 368 (2002) 70.

18 L. L. Tian, Y. H. Cheng, C. Yin, and L. B. Bai: 2015 IEEE Int. Instrumentation and Measurement Technology Conf. (I2MTC) (2015) pp. 848-852.

19 S. Xie, Z. Chen, T. Takagi, and T. Uchimoto: IEEE Trans. Magn. 47 (2011) 4582.

20 A. K. Bera and C. M. Jarque: Econ. Lett. 7 (1981) 313.

21 C. M. Chan, S. K. Tang, H. Wong, and W. L. Lee: Appl. Acoust. 73 (2012) 164. 